\title{
Expression of set is downregulated by rapamycin in human colorectal cancer cells
}

\author{
XIAOXIA WEN and YAO CHEN \\ Department of Anatomy, College of Basic and Forensic Medicine, Sichuan University, Chengdu, Sichuan 610041, P.R. China
}

Received May 16, 2013; Accepted June 04, 2013

DOI: 10.3892/br.2013.133

\begin{abstract}
The purpose of this study was to determine the mechanism through which rapamycin treatment affects the expression of the set gene in human colorectal adenocarcinoma cells. The effect of rapamycin treatment on set expression was evaluated by assessing the mRNA and protein expression of set in the SW480 and LoVo human colon carcinoma cell lines following treatment with rapamycin by quantitative polymerase chain reaction (qPCR) and western blot analysis, respectively. Our results demonstrated that the mRNA and protein levels of set were significantly decreased subsequent to rapamycin treatment in the two cell lines, indicating that set expression may be downregulated by rapamycin in human colorectal adenocarcinoma cells. Our findings suggested that the mammalian target of rapamycin signaling pathway may play a role in tumorigenesis through the regulation of the set gene.
\end{abstract}

\section{Introduction}

Colorectal adenocarcinoma is the second leading cause of malignancy-related mortality worldwide $(1,2)$, with a continuously increasing prevalence. Several factors and genes, regulating a number of pathways, are involved in colorectal tumorigenesis. Therefore, it is important to investigate the roles of these factors and genes in colorectal adenocarcinoma to promote early diagnosis, therapeutic development and cancer prevention.

A total of 86 differentially expressed sequence tags (dbESTs) from human colorectal adenocarcinoma tissues were previously identified by cDNA subtractive library construction and cDNA microarray analysis $(3,4)$. One of these dbESTs, ES274071 (GenBank accession no. NM003011.3; gene name, set) was selected for further investigation. The set gene is located on human chromosome 9p34 and encodes a

Correspondence to: Professor Yao Chen, Department of Anatomy, College of Basic and Forensic Medicine, Sichuan University, 17 Renmin Road, Chengdu, Sichuan 610041, P.R. China

E-mail: chenyao62@scu.edu.cn

Key words: human colorectal adenocarcinoma, set, mammalian target of rapamycin, rapamycin 277-amino acid protein with a molecular weight of $39 \mathrm{kDa}$. The set gene was previously found to be involved in the progression of leukemia and ovarian cancer. In a previous study by our group, quantitative reverse transcription polymerase chain reaction (qRT-PCR) analysis revealed that the mRNA level of set in colorectal cancer tissues was higher compared to that in the adjacent normal colorectal tissues, suggesting the involvement of set in the development of human colorectal adenocarcinoma (5).

The PI3K/Akt/mammalian target of rapamycin (mTOR) pathway plays a pivotal role in the development and progression of colorectal cancer by regulating cancer cell proliferation, resistance to apoptosis, angiogenesis and metastasis (6). The mTOR protein is a key kinase downstream of the growth factor receptor PI3K and the Akt signaling pathway, which are both involved in the modulation of cell growth, survival, metabolism and proliferation (7). Significant advancements have been made in elucidating the role of mTOR in cancer development and progression. Activation of the mTOR signaling pathway is often the result of genetic alterations of negative regulators of mTOR, such as phosphatase and tensin homolog (PTEN), tuberous sclerosis complex (TSC) 1 and TSC2 (8). It was demonstrated that the activation of the $\mathrm{PI} 3 \mathrm{~K} / \mathrm{Akt} / \mathrm{mTOR}$ pathway correlates with tumor progression and poor survival in a variety of tumors $(9,10)$, suggesting that mTOR is a promising molecular target for colorectal cancer therapy. The mTOR inhibitor rapamycin is a natural macrolide antibiotic isolated from Streptomyces hygroscopicus. This molecule binds to FKBP12 (FK506-binding protein) and the resulting complex inhibits the protein kinase activity of mTOR. Rapamycin was originally used as an antifungal and immunosuppressive agent. However, the subsequent identification of the inherent antiproliferative properties of rapamycin led to the investigation of this compound as a potential anticancer agent (11). Therefore, in order to elucidate the association of set with the PI3K/Akt/mTOR pathway and their role in the development and progression of colorectal cancer, the effect of rapamycin treatment on set expression in colorectal cancer cells was investigated.

\section{Materials and methods}

Cell lines and culture conditions. The SW480 and LoVo human colorectal carcinoma cell lines (obtained from the American Type Culture Collection, Manassas, VA, USA) were 
cultured in Dulbecco's modified Eagle's medium (DMEM; HyClone Laboratories, Inc., Logan, UT, USA) containing $10 \%$ fetal bovine serum, penicillin $(100 \mathrm{IU} / \mathrm{ml})$ and streptomycin $(100 \mu \mathrm{g} / \mathrm{ml})$. Cells were grown at $37^{\circ} \mathrm{C}$ in a humidified $5 \% \mathrm{CO}_{2}$ atmosphere. Experiments were performed using cells harvested from exponentially growing cultures.

Drug. Rapamycin stock solutions (5 mg/ml; Fermentek Ltd., Jerusalem, Israel) were prepared in dimethyl sulfoxide (DMSO). The solutions were stored at $-20^{\circ} \mathrm{C}$ prior to use and were diluted in DMEM to six different concentrations for subsequent experiments.

In vitro cell assays. The rapamycin stock solutions were diluted in DMEM to concentrations of $0.05,0.1,0.2,0.5,1$ and $10 \mu \mathrm{M}$. DMSO was used as the vehicle control, at a final concentration of $0.1 \%$. Cells were treated with rapamycin for $48 \mathrm{~h}$.

RNA isolation. The TRIzol reagent (Invitrogen Life Technologies, Carlsbad, CA, USA) was employed for total RNA isolation as described in the manufacturer's protocol. Total RNA yield was determined by measuring the absorbance at $260 \mathrm{~nm}$ using a spectrophotometer. The quality of RNA products was confirmed by the presence of sharp bands representing the $28 \mathrm{~S}$ and $18 \mathrm{~S}$ rRNA molecules in a $1 \%$ agarose gel, with an expected $28 \mathrm{~S}: 18 \mathrm{~S}$ intensity ratio of 2:1.

First-strand cDNA synthesis. Total RNA isolated from each sample was treated with DNase I to eliminate genomic DNA contamination prior to reverse transcription (RT). The RT reaction was performed in a $20-\mu \mathrm{l}$ volume using the $\mathrm{M}-\mathrm{MuLV}$ Reverse Transcriptase kit (Fermentas, Waltham, MA, USA) for first-strand cDNA synthesis under the recommended conditions. The synthesized cDNA product was either immediately used for qRT-PCR or stored at $-20^{\circ} \mathrm{C}$ for subsequent use.

$q R T-P C R$. The qRT-PCR reaction was performed following cDNA amplification using SYBR Premix Ex Taq (Takara Bio Inc., Shiga, Japan) and the primers were listed in Table I on a Bio-Rad C1000 real-time system (Bio-Rad, Hercules, CA, USA) according to the manufacturer's instructions and international standards (12). For each reaction, $2 \mu \mathrm{l}$ of cDNA obtained from $1 \mu \mathrm{g}$ of RNA template were used. The thermal cycling conditions were as follows: an initial denaturation step at $95^{\circ} \mathrm{C}$ for $30 \mathrm{sec}$, followed by 40 cycles of denaturation at $95^{\circ} \mathrm{C}$ for $5 \mathrm{sec}$, annealing at $62^{\circ} \mathrm{C}$ for $30 \mathrm{sec}$ and elongation at $72^{\circ} \mathrm{C}$ for $30 \mathrm{sec}$. GAPDH was used as an internal control. The amplified cDNA product was quantified using the $\Delta \Delta \mathrm{C}_{\mathrm{t}}$ method. The primers for set amplification were designed with Primer Premier 5.0 software (Premier Biosoft International, Palo Alto, CA, USA) to target its open reading frame.

Western blot assay. Cells were treated with $10 \mu \mathrm{M}$ rapamycin for $48 \mathrm{~h}$. DMSO was used as the vehicle control, at a final concentration of $0.1 \%$. Cell lysates were denatured in sample buffer containing sodium dodecyl sulphate (SDS). The same amount of denatured protein $(30 \mu \mathrm{g})$ was loaded into each lane and proteins were separated by $12 \%$ SDS-PAGE. Following this step, the proteins were transferred to PVDF membranes (Millipore, Billerica, MA, USA). After blocking for $1 \mathrm{~h}$ in
Table I. Primers.

\begin{tabular}{ll} 
Gene & \multicolumn{1}{c}{ Primer sequence $\left(5^{\prime}-3^{\prime}\right)$} \\
\hline set & F: GCTCAACTCCAACCACGAC \\
& R: TCCTCACTGGCTTGTTCATTA \\
GAPDH & F: GGAAGGTGAAGGTCGGAGT \\
& R: TGAGGTCAATGAAGGGGTC
\end{tabular}

Tris-buffered saline containing $0.1 \%$ Tween-20 and $3 \%$ bovine serum albumin, the membranes were incubated overnight at $4^{\circ} \mathrm{C}$ with an anti-SET primary antibody (1:200; Santa Cruz Biotechnology, Inc., Santa Cruz, CA, USA). The membranes were then incubated with an appropriate horseradish peroxidase-conjugated secondary antibody and the corresponding protein product was visualized using ECL reagent (Thermo Fisher Scientific, Waltham, MA, USA).

Statistical analysis. Statistical analysis was performed using the t-test with SPSS software, version 19.0 (SPSS Inc., Chicago, IL, USA). $\mathrm{P}<0.05$ was considered to indicate a statistically significant difference.

\section{Results}

As shown in Fig. 1, the set mRNA expression decreased with the increasing concentration of rapamycin, as compared to cells treated with DMSO alone. In SW480 cells, the set mRNA expression levels relative to the control DMSO were 80.46, $80.18,75.85,69.95,57.2$ and $49.65 \%$, for rapamycin concentrations ranging from 0.05 to $10 \mu \mathrm{M}$. In this case, only the downregulation in expression observed with the two highest concentrations ( 1 and $10 \mu \mathrm{M}$ ) were considered statistically significant according to the t-test analysis $(\mathrm{P}=0.033)$. The corresponding values in LoVo cells were 93.8, 91.41, 89.18, $78.73,68.43$ and $50.35 \%$, respectively; for this cell type, only the downregulation in response to the highest concentration $(10 \mu \mathrm{M})$ was considered statistically significant according to the t-test analysis $(\mathrm{P}=0.027)$.

As shown in Fig. 2, following treatment with $10 \mu \mathrm{M}$ rapamycin for $48 \mathrm{~h}$, the SET protein expression was downregulated in SW480 and LoVo cells treated with rapamycin, as compared to the cells treated with the vehicle control. The relative expression levels of SET were $46.45 \%(\mathrm{P}=0.023)$ and $39.93 \%$, respectively $(\mathrm{P}=0.022)$.

\section{Discussion}

In a previous study, qPCR analysis demonstrated that set gene expression was significantly upregulated in human colorectal adenocarcinoma tissues, as compared to adjacent normal colorectal tissues. Although there was no significant correlation between set gene expression and patient characteristics such as gender, age, Dukes' stage, or tumor differentiation, a higher rate was observed in Dukes' C (70.59\%) and D stages $(71.43 \%)$ compared to Dukes' B stage $(50 \%)$. This observation was in accordance with the findings of Ouellet on human ovarian cancer (13). The inhibition of set gene expression effectively 

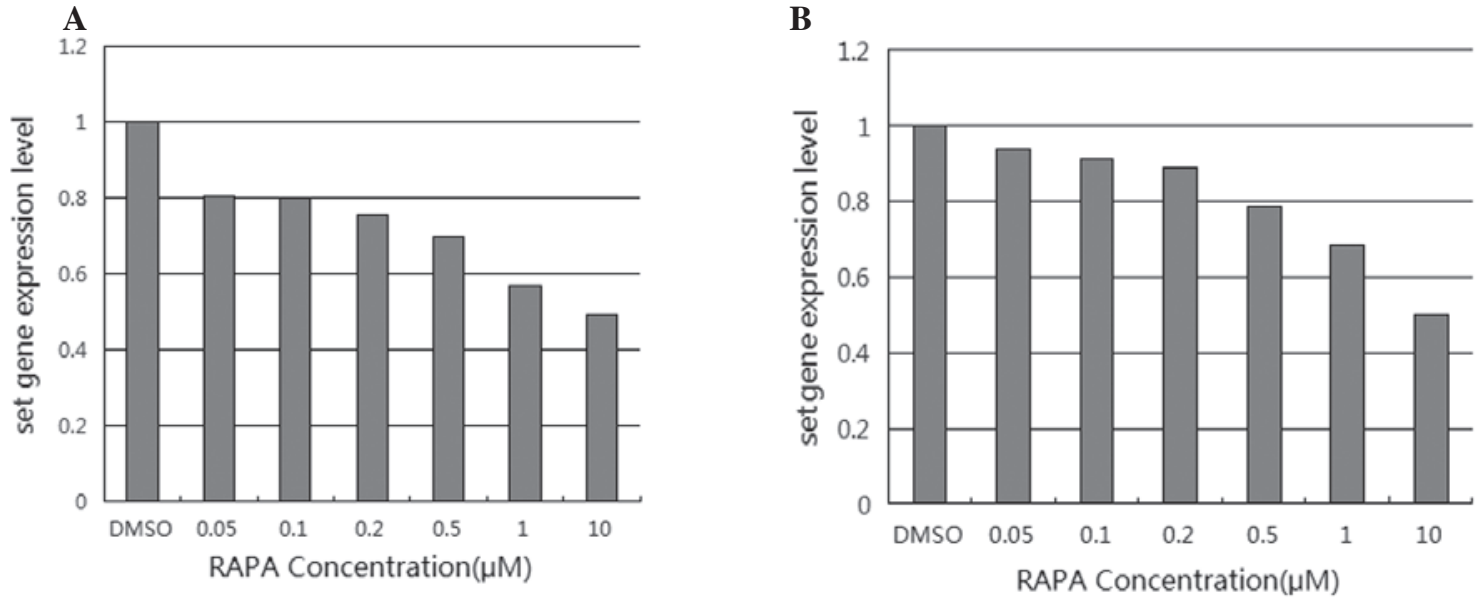

Figure 1. set mRNA expression in human colorectal carcinoma cell lines following a 48-h rapamycin treatment. (A) set mRNA expression in SW480 cells following treatment with different concentrations of rapamycin, ranging from 0 to $10 \mu \mathrm{M}$. (B) set mRNA expression in LoVo cells following treatment with different concentrations of rapamycin, ranging from 0 to $10 \mu \mathrm{M}$. RARA, rapamycin; DMSO, dimethyl sulfoxide.

A
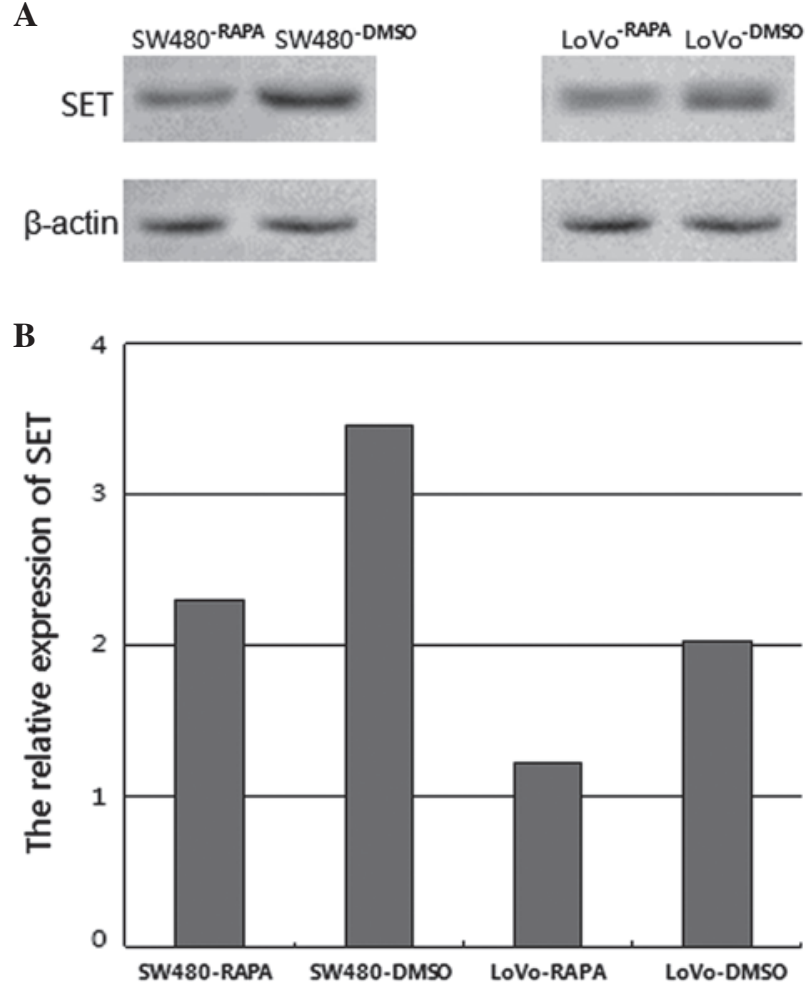

Figure 2. (A) SET protein expression in human colorectal carcinoma cell lines following treatment with $10 \mu \mathrm{M}$ rapamycin for $48 \mathrm{~h}$. Lane 1 , SW480 cells treated with rapamycin; lane 2, SW480 cells treated with DMSO; lane 3, LoVo cells treated with rapamycin; and lane 4, LoVo cells treated with DMSO. (B) Quantification of protein bands from western blot analysis. SET expression is upregulated in SW480 and LoVo cells treated with rapamycin, compared to the corresponding DMSO-treated control cells. RARA, rapamycin; DMSO, dimethyl sulfoxide.

suppressed cell proliferation and promoted cell apoptosis, suggesting that the set gene is involved in the development of human colorectal adenocarcinoma (5). In the present study, we demonstrated that rapamycin treatment was able to induce set downregulation in human colorectal adenocarcinoma cell lines at the mRNA and protein levels.
The set gene was initially identified by von Lindern et al (14) in a patient with acute undifferentiated leukemia. A translocation of chromosome 9 resulted in the generation of the SET-CAN fusion protein. The set gene is crucial in the progression of acute undifferentiated leukemia, possibly by activating $\mathrm{CAN}$ in the nucleus and stimulating the transformation potential of SET-CAN. Cristóbal et al (15) suggested that set overexpression may be a key mechanism responsible for the inhibition of protein phosphatase $2 \mathrm{~A}$ in acute myeloid leukemia. Almeida et al (16) assessed the effect of the overexpression of the SET protein, a histone acetylation modulator accumulated in head and neck squamous cell carcinoma, on the gene regulation and protein activity of aldehyde dehydrogenase 2 and glutathione S-transferase P1. Leopoldino et al (17) reported that accumulated set may act via the Akt/PTEN pathway as a cell survival signal or as an oxidative stress sensor promoting cell death. The studies mentioned above indicated that set may be involved in the development of several malignant tumors.

The mTOR protein is a serine/threonine protein kinase involved in a nutrient-sensitive signaling pathway that is crucial in regulating cell growth and proliferation. The mTOR pathway is activated in various cell processes, including tumorigenesis, insulin resistance, adipogenesis, angiogenesis and T-lymphocyte activation. In addition, the mTOR pathway is associated with various human diseases, such as cancer, obesity and type 2 diabetes (7). The activation of the PI3K/Akt/mTOR pathway inhibits apoptosis induced by several types of stimuli, thereby promoting cell cycle progression, cell survival and proliferation, which are important for tumor invasion and metastasis. Moreover, the role of the PI3K/Akt/mTOR pathway in neovascularization may be another mechanism through which it promotes tumorigenesis. The PI3K/Akt/mTOR pathway was also found to be involved in reactive oxygen species-induced and integrin $\beta 3$-mediated migration and invasion of colorectal cancer cells (18). Kim et al (19) reviewed the key components of the $\mathrm{PI} 3 \mathrm{~K} / \mathrm{Akt} / \mathrm{mTOR}$ pathway, their molecular alterations and the inhibitors targeting the mTOR pathway in colorectal cancer.

It was reported that rapamycin is able to induce apoptosis, suggesting a potential role of the mTOR pathway in 
regulating cell survival (20). Moreover, rapamycin has been shown to be effective in the clinical treatment of several types of cancer. For example, Boffa et al (21) demonstrated that rapamycin treatment may inhibit cancer cell growth and metastatic progression of non-small-cell lung carcinoma. Samkari et al (22) demonstrated that rapamycin treatment may induce the expression of the anti-apoptotic protein survivin in neuroblastoma cells. Kim et al (19) suggested that mTOR inhibitors may be attractive antitumor drugs, which may be used in combination with other treatment strategies.

In the present study, we demonstrated that rapamycin treatment was able to induce set downregulation in the SW480 and LoVo human colorectal adenocarcinoma cell lines at the mRNA and protein levels. The downregulation of set mRNA expression was observed with rapamycin treatment at all six tested concentrations, ranging from 0.05 to $10 \mu \mathrm{M}$. However, in SW480 cells, only the downregulation in response to the highest concentrations (1 and $10 \mu \mathrm{M}$ ) was considered statistically significant. The same effect was observed in LoVo cells, with only the downregulation in response to the highest concentration $(10 \mu \mathrm{M})$ being statistically significant. Thus, the effective concentration of rapamycin for significant set downregulation was $10 \mu \mathrm{M}$ in the two cell lines. In the present study, we have demonstrated that rapamycin was able to inhibit the expression of the set gene in human colorectal adenocarcinoma cell lines. The dose-dependent association between set gene expression and rapamycin treatment suggests the potential involvement of set in the PI3K/Akt/mTOR pathway in colorectal adenocarcinoma cells, possibly through the regulation of mTOR activity. The set gene may act as an oncogene in colorectal adenocarcinoma by inhibiting cell apoptosis, accelerating cell cycle progression and promoting metastasis through the activation of the PI3K/Akt/mTOR pathway, which is opposite to the effects of rapamycin. Furthermore, the downregulation of set by rapamycin treatment suggests that rapamycin may be used as an antineoplastic drug in colorectal adenocarcinoma therapy. The PI3K/Akt/mTOR pathway may be a diagnostic marker and molecular target in colorectal adenocarcinoma. However, further investigation is required to comprehensively analyze the functions of set and the PI3K/Akt/mTOR pathway in carcinogenesis.

\section{Acknowledgements}

This study was supported by the National Natural Science Foundation of China (grant no. 81072023).

\section{References}

1. Jemal A, Bray F, Center MM, Ferlay J, Ward E and Forman D: Global cancer statistics. CA Cancer J Clin 61: 69-90, 2011.

2. Herrinton LJ, Liu L, Levin TR, Allison JE, Lewis JD and Velayos F: Incidence and mortality of colorectal adenocarcinoma in persons with inflammatory bowel disease from 1998 to 2010 Gastroenterology 143: 382-389, 2012.
3. Chen Y, Zhang Y, Zhou Z, Wang G and Yi Z: Identification of differentially expressed genes in human colorectal adenocarcinoma. World J Gastroenterol 12: 1025-1032, 2006.

4. Zhang $\mathrm{C}$ and Chen Y: Electronic cloning and validating of the suppression subtractive hybridization EST ES274070 of human colorectal adenocarcinoma. US Chin J Lymphol Oncol 6: 83-88, 2007.

5. Jiang Q, Zhang C, Zhu J, Chen Q and Chen Y: The set gene is a potential oncogene in human colorectal adenocarcinoma and oral squamous cell carcinoma. Mol Med Rep 4: 993-999, 2011.

6. Zoncu R, Efeyan A and Sabatini DM: mTOR: from growth signal integration to cancer, diabetes and ageing. Nat Rev Mol Cell Biol 12: 21-35, 2011.

7. Laplante M and Sabatini DM: mTOR signaling at a glance. J Cell Sci 122: 3589-3594, 2009.

8. Feng Z, Zhang H, Levine AJ and Jin S: The coordinate regulation of the p53 and mTOR pathways in cells. Proc Natl Acad Sci USA 102: 8204-8209, 2005.

9. Gulhati P, Cai Q, Li J, et al: Targeted inhibition of mammalian target of rapamycin signaling inhibits tumorigenesis of colorectal cancer. Clin Cancer Res 15: 7207-7216, 2009.

10. Chiang GG and Abraham RT: Targeting the mTOR signaling network in cancer. Trends Mol Med 13: 433-442, 2007.

11. Miyake N, Chikumi H, Takata M, Nakamoto M, Igishi T and Shimizu E: Rapamycin induces p53-independent apoptosis through the mitochondrial pathway in non-small cell lung cancer cells. Oncol Rep 28: 848-854, 2012.

12. Bustin SA, Benes V, Garson JA, Hellemans J, et al: The MIQE guidelines: minimum information for publication of quantitative real-time PCR experiments. Clin Chem 55: 611-622, 2009.

13. Ouellet V, Le Page C, Guyot MC, Lussier C, Tonin PN, Provencher DM and Mes-Masson AM: SET complex in serous epithelial ovarian cancer. Int J Cancer 119: 2119-2126, 2006.

14. von Lindern M, Van Baal S, Wiegant J, Raap A, Hagemeijer A and Grosveld G: Can, a putative oncogene associated with myeloid leukemogenesis, may be activated by fusion of its 3 half to different genes: characterization of the set gene. Mol Cell Biol 12: 3346-3355, 1992.

15. Cristóbal I, Garcia-Orti L, Cirauqui C, Cortes-Lavaud X, García-Sánchez MA, Calasanz MJ and Odero MD: Overexpression of SET is a recurrent event associated with poor outcome and contributes to protein phosphatase $2 \mathrm{~A}$ inhibition in acute myeloid leukemia. Haematologica 97: 543-550, 2012.

16. Almeida LO, Goto RN, Pestana CR, Uyemura SA, Gutkind S, Curti $\mathrm{C}$ and Leopoldino AM: SET overexpression decreases cell detoxification efficiency: ALDH2 and GSTP1 are downregulated, DDR is impaired and DNA damage accumulates. FEBS J 279: 4615-4628, 2012.

17. Leopoldino AM, Squarize CH, Garcia CB, et al: Accumulation of the SET protein in HEK293T cells and mild oxidative stress: cell survival or death signaling. Mol Cell Biochem 363: 65-74, 2012.

18. Lei Y, Huang K, Gao C, et al: Proteomics identification of ITGB3 as a key regulator in reactive oxygen species-induced migration and invasion of colorectal cancer cells. Mol Cell Proteomics 10: M110.005397, 2011.

19. Kim DD and Eng C: The promise of mTOR inhibitors in the treatment of colorectal cancer. Expert Opin Investig Drugs 21: 1775-1788, 2012.

20. Thimmaiah KN, Easton J, Huang S, Veverka KA, Germain GS, Harwood FC and Houghton PJ: Insulin-like growth factor I-mediated protection from rapamycin-induced apoptosis is independent of Ras-Erk1-Erk2 and phosphatidylinositol 3'-kinase-Akt signaling pathways. Cancer Res 63: 364-374, 2003.

21. Boffa DJ, Luan F, Thomas D, Yang h, Sharma VK, Lagman M and Suthanthiran M: Rapamycin inhibits the growth and metastatic progression of non-small cell lung cancer. Clin Cancer Res 10: 293-300, 2004.

22. Samkari A, Cooper ZA, Holloway MP, Liu J and Altura RA: Rapamycin induces the anti-apoptotic protein survivin in neuroblastoma. Int J Biochem Mol Biol 3: 28-35, 2012. 